\title{
Gain Improvement of Microstrip Patch Antenna Using Negative Permeability Metamaterial Reflecting Surface
}

\author{
Atul kumar ${ }^{1}$, Nitin Kumar ${ }^{2}$, Dr. S.C Gupta ${ }^{2}$ \\ ${ }^{1}$ (PG Student, M-Tech digital communication, DIT Dehradun, India) ${ }^{2}$ (Department of ECE,DIT Dehradun,India)
}

\begin{abstract}
This methodology involves complementary square split ring resonators and a microstrip patch antenna both designed to resonate at same frquency. The proposed antenna is made by using metamaterial reflecting surface to obtain the maximum gain hight of reflecting surface is adjusted.we uses an array of squared split ring resonator (SRR) printed on a low dielectric constant material as the MRS, which is suspended over a probe-fed patch antenna. HFSS simulation of the proposed antenna shows improved gain and return loss at the centre frequency of $4.72 \mathrm{GHz}$.By using metamaterial reflecting surface technique we obtain $4.87 \mathrm{db}$ gain improvement.
\end{abstract}

Keywords:Microstrip patch antenna, SRR, Metamaterials, HFSS

\section{Introduction}

Patch antennas play a very important role in today's world of wireless communication systems as these cover a broad range of frequencies. These patch antennas are simple and are used for the most demanding applications. Low weight, low fabrication cost, circular polarizations, dual band operation, frequency agility, feed line ${ }^{\circ}$ exibility, beam scanning are a few notable advantages of microstrip patch antenna. Even though there are many advantages a few critical drawbacks which limit the applicability of microstrip patch antennas are low efficiency, low gain, narrow bandwidth,low power handling capability. There are a lot of techniques to improve on the antenna parameters like defected ground structures, parasitic elements or slots, thick substrate with lowpermittivity, stacked patches [1]. Meta-materials can also be used to enhance the antenna parameters . Amongst techniques for gain enhancement, one novel method is to include metamaterial onto antenna's superstrate. In recent years, a subgroup of the family of superstrate antennas that have attracted considerable interest is the partially reflecting surface (PRS) antennas. The PRS is generally realized using a planar periodic material (patch or strip) similar to a frequency selective surface (FSS) [2-3]. By replacing a PRS with a metamaterial, which is designated as metamaterial reflecting surface (MRS), the profile of the MRS antenna can be significantly reduced. Properties and applications of metamaterial structure based antennas have been particularly considered for high-gain applications [4-5]. Furthermore, by properly selecting the thicknesses of the substrate and superstrate layers and by shaping the MRS, a very high gain can be realized.

\section{Design Of Negative Permeability Metamaterial}

To explain the electromagnetic properties of the negative permeabilitymaterial,improved retrievel parameter method based on $\mathrm{S}$ parameter[6] or $\mathrm{n}$ and $\mathrm{z}$ relationis given by this formula

$$
\varepsilon=n / z, \quad \mu=n z
$$

where $\mathrm{n}$ is refractive index and $\mathrm{z}$ is the wave impedence of the slab.

According to Dr. smith [7][8] we can write $\mathrm{n}$ and $\mathrm{z}$ as follow

$$
\begin{aligned}
& \mathrm{n}=(1 / \mathrm{kd}) \cos ^{-}\left[1 / 2 \mathrm{~S}_{21}\left(1-\mathrm{S}_{11}{ }^{2}+\mathrm{S}_{21}{ }^{2}\right)\right] \\
& \mathrm{Z}= \pm\left[\left(\left(1+\mathrm{S}_{11}\right)^{2}-\mathrm{S}_{21}{ }^{2}\right) /\left(\left(1-\mathrm{S}_{11}\right)^{2}-\mathrm{S}_{21}{ }^{2}\right)\right]^{1 / 2}
\end{aligned}
$$

For passive material $\mathrm{im}(\mathrm{n}) \geq 0$ and $\mathrm{re}(\mathrm{z}) \geq 0$

\section{Negative Permeability Metamaterial}

The Split ring resonator (SRR) is a metamaterial sample which was proposed by pendry[9].SRR is a ring with gap,and axis of the ring should be parallel with the magnetic fieldat the resonance frequency band the permeability is negative. 


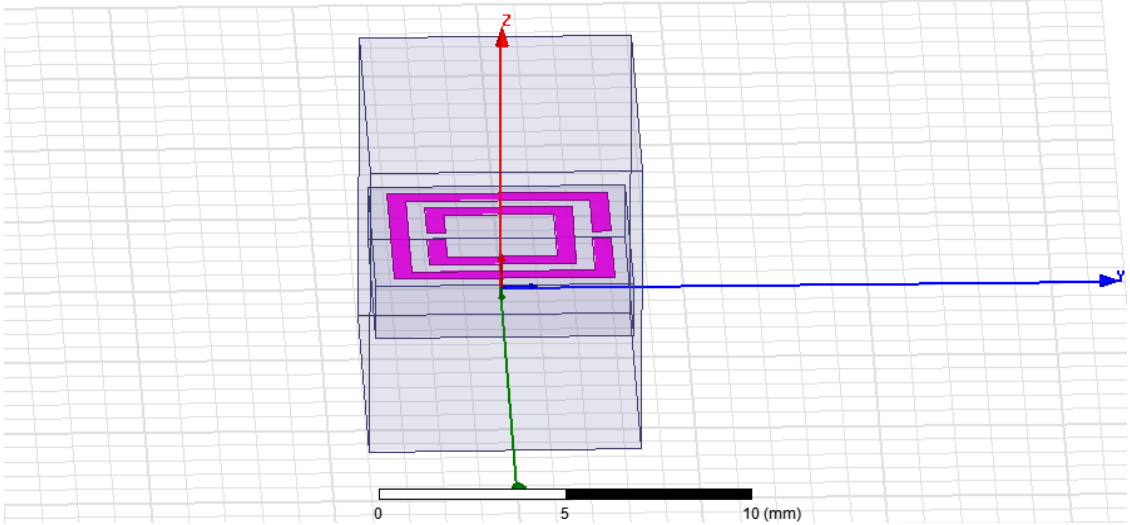

Fig 1.Strucure of $\mu$ negative metamaterial

Inside squre split ring is $4 \mathrm{~mm}$ and size of outsidesqure ring is $6 \mathrm{~mm}$, split gap and sepration distance between split ring is $0.5 \mathrm{~mm}$ and width of split ring is also $0.5 \mathrm{~mm}$. This split ring are paste on a substrate duroid tm relative permittivity 2.2 which hight is $1.5 \mathrm{~mm}$. And position or size of air gap is as $\quad(-4.1,-2.7,-2.9 \mathrm{~mm})$ and $\mathrm{X}$ $, \mathrm{Y}, \mathrm{Z}$ size $9.6,7.4,8.1 \mathrm{~mm}$. This structure will show all properties of negative permeability metamaterial.This negative permeability metamaterial resonant at $4.72 \mathrm{ghz}$.

Fig. 2 and fig. 3 show the simulated parameters of split ring resonator here we can see permeability resonant at $4.72 \mathrm{ghz}$ and have negative from 4.72 to $5 \mathrm{ghz}$,and fig. 3 show the s parameters.

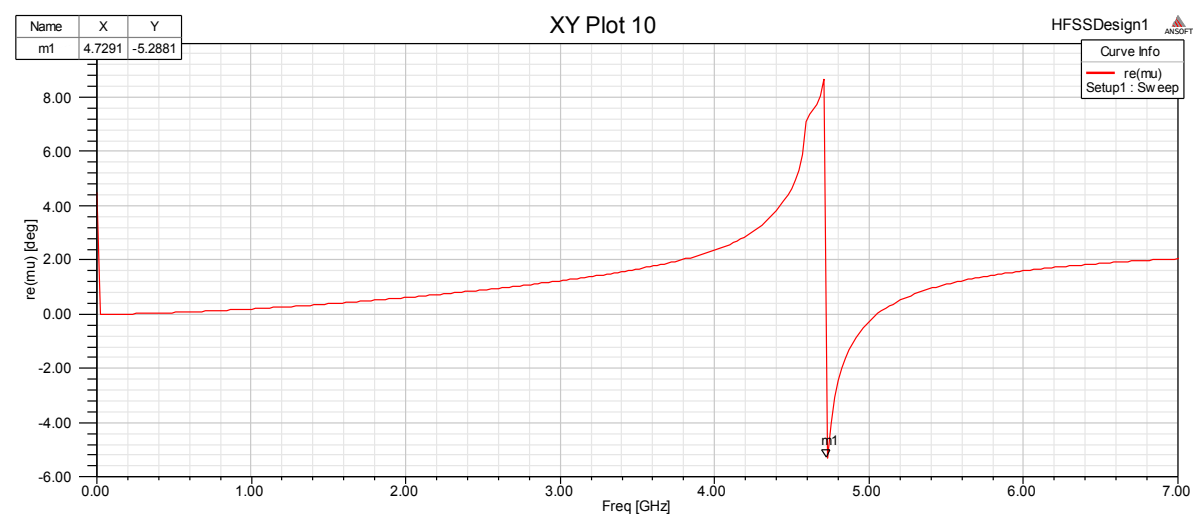

Fig 2.Effective permeability SRR

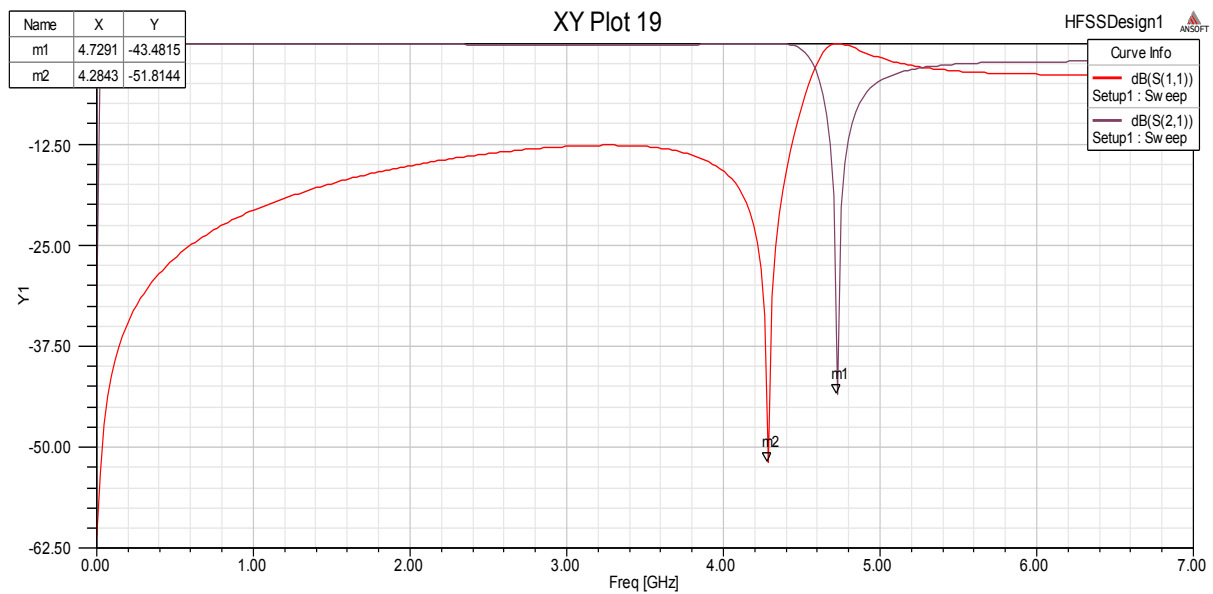

Fig 3.Simulated S parameters of SRR

IV. Microstrip Patch Antenna With Metamaterial Reflecting Surface

The proposed antenna geometry and configuration is depicted in Figure 4, where a threelayerstructure consists of a substrate, air-gap, and a superstrate of thicknesses $h 1, h 2$, and $h 3$, respectively. The antenna is designed to be operated at $4.72 \mathrm{GHz}$. The antenna is composed of a $20.58 \times 25.12 \mathrm{~mm} 2$ copper patch printed on 
a duroid substrate that has a dielectric constant ( $(\mathrm{r} 1)$ of 2.2 , a thickness (h1) of $1.5 \mathrm{~mm}$ and an area of $60 \times 60$ $\mathrm{mm} 2$. The patch isexcited by a $50-\Omega$ probe feed. The MRS superstrate is built on a $0.763 \mathrm{~mm}$ thick (h3) dielectricmaterial with a dielectric constant ( $(\mathrm{r} 2)$ of 3.2. The superstrate is supported by foams so that it keepsan air-gap above the substrate, vary the superstratehight to obtain the maximum gain. The MRS is composed of $8 \times 8$ unit cells. Each cell has a spacing of $1 \mathrm{~mm}$ in $x$ and $y$-direction to the adjacent ones as shown in Figure 1 . Probe feed is given by copper strip of $10 \times 30 \mathrm{~mm}^{2}$ at reflecting surface. The resonant condition can be satisfied byadjusting the MRS superstrate configuration including dimensions of unit-cell and grid sizes. The maximum gain at the resonant frequency is a function of the superstrate thickness. Therefore, there is a compromise amongst the gain improvement, impedance matching and the overall height of theantenna.

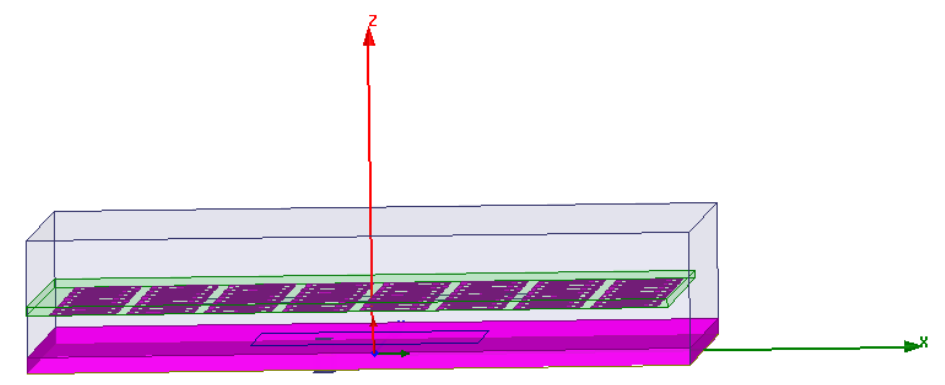

Fig 4.Configuration of the microstrip patch antenna with a metamaterial reflecting surface

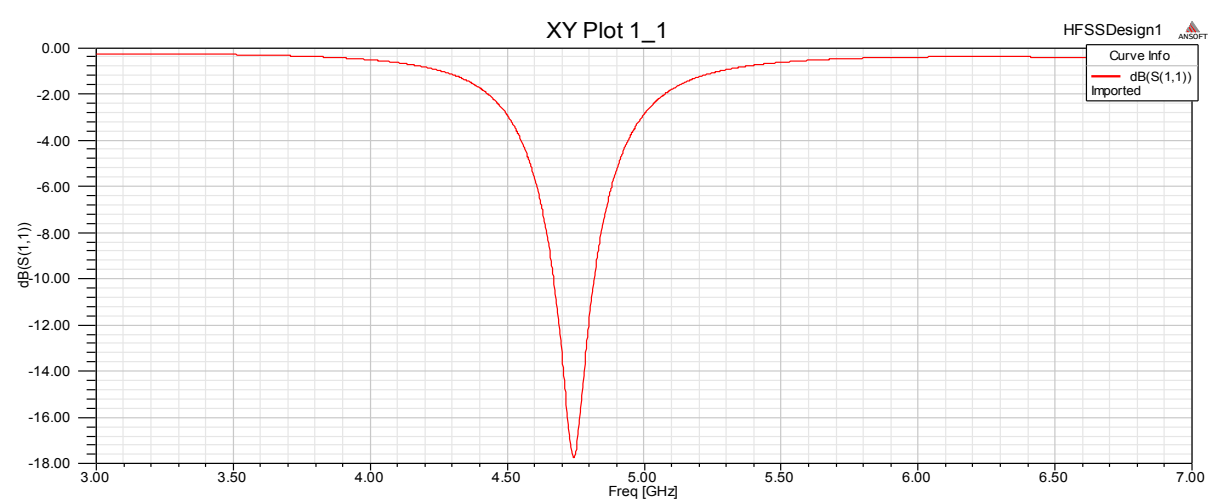

Fig 5.S Parameter of simple patch antenna at $4.72 \mathrm{GHZ}$

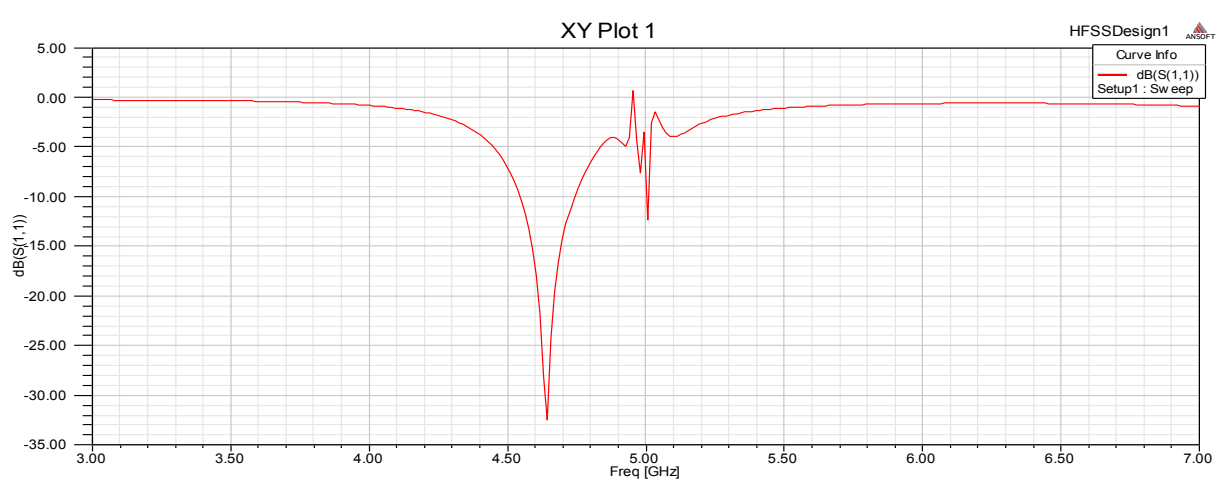

Fig 6.S Parameter of patch antenna loaded with mu negative metamaterial

Fig . 5 show the simulated s parameters of patch antenna at resonance frequency $4.72 \mathrm{GHZ}$ and fig 6 show the S- parameters of antenna with metamaterial reflecting surface.return loss with $\mu$ negative is $-33 \mathrm{db}$ as compare to $-18 \mathrm{db}$ without metamaterial.we can see gain improvement is about $4.87 \mathrm{db}$ from fig 7 and 8 . And Beam width can be reduced by 14 degree. 

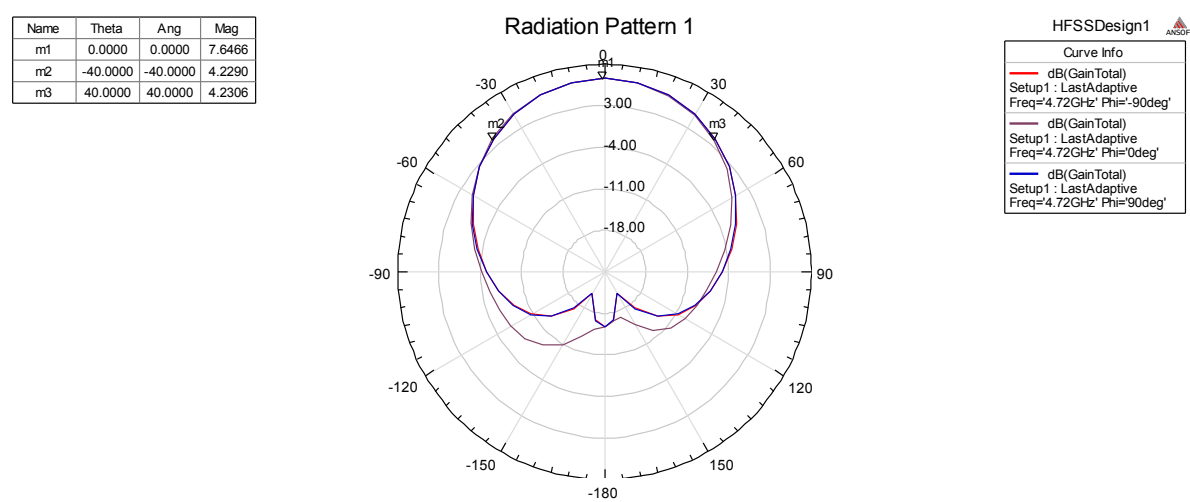

Fig 7. Gain of Microstrip patch antenna
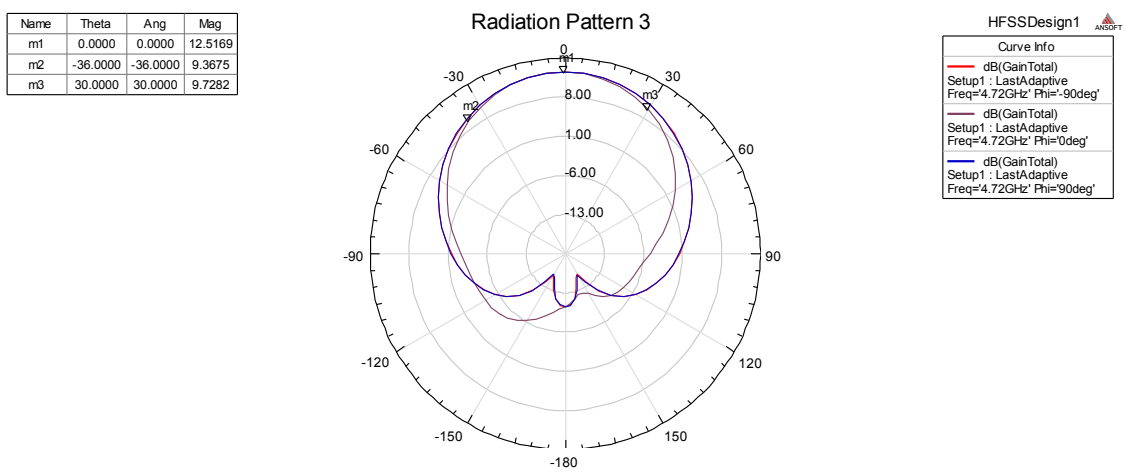

Fig 8. Gain of patch antenna with a metamaterial reflecting surface

\section{Conclusion}

This paper presents a low-cost high-gain microstrip patch antenna for 4.72-GHz WLANapplications. The proposed antenna employs a metamaterial reflecting surface (MRS) as thesuperstrate for the gain improvement. Unlike the use of conventional PRS,. We havemeasured the gain of the MRS antenna and confirmed that a considerable gain improvement of $4.87 \mathrm{~dB}$ has been obtained over a conventional patch-only case. Measured and simulated results havebeen presented with good agreement.

\section{Refrences}

[1]. Lee, K.-F. and K.-F. Tong, Microstrip patch antenna-basic characteristics and some recentadvances," Proceedings of the IEEE, Vol. 100, No. 7, Jul. 2012.

[2]. D. H. Lee, Y. J. Lee, R. Mittra, W. S. Park, "Design of novel thin frequency selective surface superstrates for dual-band directivity enhancement”, IET Microw. Antennas Propag., vol. 1, No.1, pp. 248-254, Feb. 2007.

[3]. A. R. Weily, T. S. Bird, Y. J. Guo "A reconfigurable high-gain partially reflecting surface antenna”, IEEE Trans. Antennas Propag., vol. 56, No. 1, pp. 3382-3390, Nov. 2008.

[4]. S. Wang, A. P. Feresidis, G. Goussetis, J. C. Vardaxoglou, "High-gain subwavelength resonant cavity antennas based on metamaterialgorund planes", IEE Proc.-Microw. Antennas Propag.,vol. 153, No. 1, pp. 1-6, Feb. 2006.

[5]. J. Ju, D. Kim, W. J. Lee, J. I. Choi, "Wideband high-gain antenna using metamaterialsuperstrate with the zero reflactive index", Microw. Opt. Technol. Lett., vol. 51, No. 8, Aus. 2009.

[6]. MAO xuTONG DUAN Zhao- yun Wang yanshuai" an improved parameters retrievel method" journal of microwave aug2010.

[7]. D.r Smith S.SchultzP.Marko "Determination of effective permeability and permettivity from reflection and transmission coefficient “" phys .Rev. b 2002, 65: 195104.

[8]. D.R Smith, D, C Vier, C, M soukoulis.”Electromagnetic parameter retrieval from inhomogenousmetamaterials” Phys Rev .b 2005 71: 036617.

[9]. J.B. Pendry, A.J. Holden, D.J. Robbins, W.J. Stewart, "magnetism from conductors and enhanced nonlinear phenomena" IEEE Trans. Micro Tech. vol.47 no.11, pp.2075-2081, Nov.1999. 